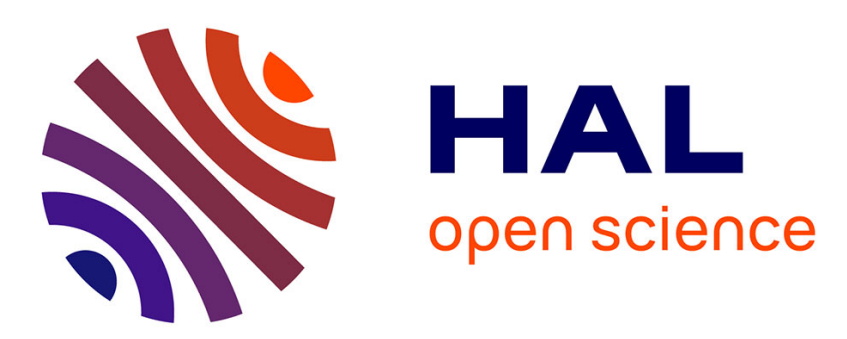

\title{
FORMATION DES CENTRES F, M ET R DANS LE FLUORURE DE LITHIUM
}

\author{
P. Durand, Y. Farge, M. Lambert, R. Smoluchowski
}

\section{To cite this version:}

P. Durand, Y. Farge, M. Lambert, R. Smoluchowski. FORMATION DES CENTRES F, M ET R DANS LE FLUORURE DE LITHIUM. Journal de Physique Colloques, 1967, 28 (C4), pp.C4-154-C4157. 10.1051/jphyscol:1967426 . jpa-00213313

\section{HAL Id: jpa-00213313 https://hal.science/jpa-00213313}

Submitted on 1 Jan 1967

HAL is a multi-disciplinary open access archive for the deposit and dissemination of scientific research documents, whether they are published or not. The documents may come from teaching and research institutions in France or abroad, or from public or private research centers.
L'archive ouverte pluridisciplinaire HAL, est destinée au dépôt et à la diffusion de documents scientifiques de niveau recherche, publiés ou non, émanant des établissements d'enseignement et de recherche français ou étrangers, des laboratoires publics ou privés. 


\title{
FORMATION DES CENTRES $F, M$ ET $R$ DANS LE FLUORURE DE LITHIUM ${ }^{(1)}$
}

\author{
P. DuRand, Y. Farge, M. Lambert et R. Smoluchowski ( ${ }^{2}$ ) \\ Service de Physique des Solides $\left({ }^{3}\right.$ ), Faculté des Sciences, 91-Orsay (France)
}

\begin{abstract}
Résumé. - La formation des centres $M$ et $R$ dans LiF eșt précédée par l'apparition de centres $F_{2}^{+}$et $F_{3}^{+}$formes par diffusion à une température voisine de l'ambiante, de centres $\alpha$ vers des centres $F$ et $M$. Le nombre de centres $F$ varic proportionnellement à la racine carrée de la dose d'irradiation, la constante de proportionnalité étant une fonction décroissante de la temperature.
\end{abstract}

Abstract. - The formation of $M$ and $R$ centers in $L F$ is preceded by the formation of $F_{2}^{+}$ and $F_{\bar{y}}$ centers out of $\alpha$ and $F, \alpha$ and $M$ centers at temperatures near room temperature. The number of $F$ centers is then proportional to the square root of the radiation time, the constant being a decreasing function of temperature.

L'irradiation des halogénures alcalins par les rayonnements ionisants (rayons $X$. électrons) crée essentiellement des défauts dans le réscau des ions halogènes [1] : lacunes et interstitiels ayant ou non capté un électron (ccntres $\alpha$ et $F$, centres $H$ et $H^{\prime}$ ). Suivant la température de l'irradiation ces défauts primaires sont plus ou moins stables et plus ou moins mobiles; audessus de $77^{\circ} \mathrm{K}$, les centres $\alpha$ sont peu nombreux [2] Ies interstitiels, s'ils existent, se trouvent dans un état différent des centres $H$ et $H^{r}$ [3] et deviennent probablement assez mobiles. Les centres $F$ prédominent et si la température est suffisamment élevée, les centres complexes $(M, R)$ apparaissent. Nous reporterons ici les résultats concernant :

1) la formation des centres complexes soit par recuit de cristaux irradiés à basse température, soit par irradiation directe à une température suffisamment élevée : dans les deux cas le mécanisme est sensiblement le même et implique l'existence ct la mobilité des centres $\alpha$ à la température où se forment les centres complexes [4];

2) la loi de création des centres $F$ qui varie elie aussi avec la température de l'itradiation.

(1) Cet atticle recouvre en partie le travail de la thèse de doctorat ès sciences physiques, enregistree au C. N. R. S. sous le $n^{\circ} \mathrm{AO} 1427$, et qui sera soutenue par Y. Farge à la Faculté des Sciences d'Orsay en 1967.

(2) Adresse pemanente : University of Princeton, Princeton, New Jersey, U. S. A.

(3) Laboratoire associes au C. N. R. S.
Tontes les expériences ont été faites en utilisant des cristaux provenant de la compagnie Harshaw et contenant un taux d'impuretés inférieur à $5 \mathrm{p}$. p. m. Ils ont été irradiés soit aux électrons soit aux rayons $\mathrm{X}$ et à des températures comprises entre $77^{\circ} \mathrm{K}$, et $573^{\circ} \mathrm{K}$.

I. Formation des centres complexes : $\boldsymbol{M}$ et $\boldsymbol{R}$. Dans un article précédent [5] nous avons montré que les cristaux de fluorure de lithium irradiés pouvaient contenir, non seulement les centres complexes $M\left(F_{2}\right)$ et $R\left(F_{3}\right)$, mais également les centres ionisés : $F_{2}^{+}$et $F_{3}^{+}$ dont nous avons identifié les bandes d'absorption et d'émission :

$$
F_{2}^{+}\left\{\begin{array} { l } 
{ 6 4 5 0 \AA } \\
{ 9 0 0 0 \AA }
\end{array} \quad F _ { 3 } ^ { + } \left\{\begin{array}{l}
4500 \AA \\
5300 \AA
\end{array}\right.\right.
$$

La détection de ces centres est donc relativement facile; la seule difficulté, dans le cas des centres $F_{3}^{+}$, réside dans le fait que la bande d'absorption est confondue aves celle des centres $M$, toujours plus intense : les contres $F_{3}^{+}$doivent donc toujours être observés au moyen de leur émission.

Cette identification des centres $F_{2}^{+}$et $F_{3}^{+}$nous a permis de montrer qu'ils apparaissent toujours comme intermédiaires respectivement dans la formation des centres $F_{2}$ et $F_{3}$. Lorsque les cristaux de fluorure de lithium sont irradiés aux électrons de $1,4 \mathrm{MeV}$ $\left(3 \times 10^{15}\right.$ électrons $\left./ \mathrm{cm}^{2}\right)$ à $2488^{\circ} \mathrm{K}$ et soumis à des recuits isochrones de $13 \mathrm{mn}$ à des températures croissantes on voit se former successivemant les centras $F_{2}^{+}$ 


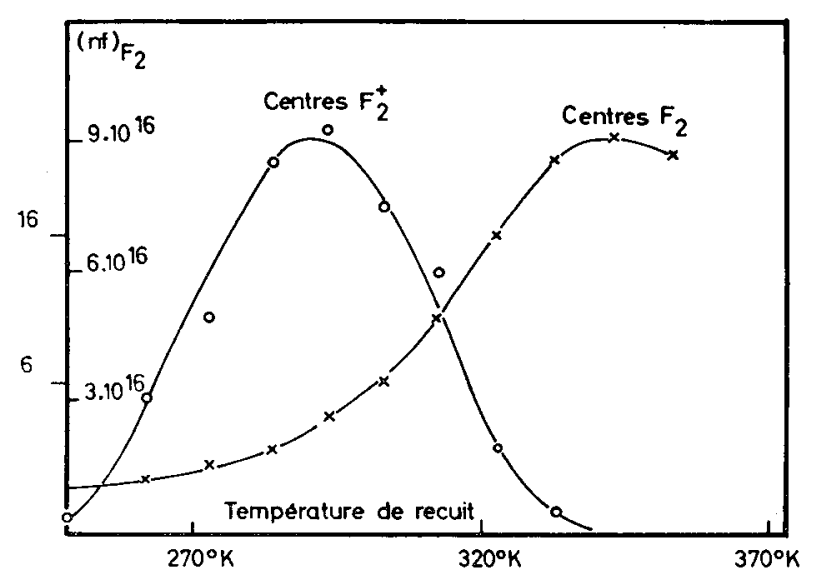

FIG. 1. - Variation avec la température de recuit du nombre de centres $F_{2}^{+}$et $F_{2}$ (aux forces d'oscillateur près), au cours de recuits isochrones d'une durée de 13 minutes dans un cristal de LiF, irradié aux électrons $\left(3 \times 10^{15} \mathrm{e} / \mathrm{cm}^{2} ; 1,4 \mathrm{MeV}\right)$ à $248^{\circ} \mathrm{K}$.

puis $F_{2}$ (Fig. 1). Les centres $F_{2}^{+}$n'existent qu'au moment où les centres $F_{2}$ se forment. L'évolution relative des centres $F_{3}^{+}$et $F_{3}$ est plus complexe et se trouve décrite par ailleurs [6]. Nos résultats sont en accord avec le mécanisme proposé par Delbecq [7] : diffusion d'un centre $\alpha$ vers un centre $F$ (ou $F_{2}$ ), puis capture d'un électron par le centre $F_{2}^{+}\left(\right.$ou $F_{3}^{+}$) ainsi formé :

$$
\left\{\begin{array}{l}
F+\alpha \rightarrow F_{2}^{+} \\
F_{2}^{+}+\mathrm{e} \rightarrow F_{2}
\end{array}\right.
$$

$$
\left\{\begin{array}{l}
F_{2}+\alpha \rightarrow F_{3}^{+} \\
F_{3}^{+}+\mathrm{e} \rightarrow F_{3}
\end{array}\right.
$$

Des études cinétiques à température constante nous ont permis :

- de vérifier que le nombre de centres $F_{2}$ formés croît bien comme le nombre de centres $F_{2}^{+}$disparus;

- de déterminer l'énergie d'activation de diffusion du centre $\alpha$ : les valeurs expérimentales ne sont pas les mêmes dans les cas (1) et (2). Nous avons trouvé respectivement : $0,67 \mathrm{eV}$ et $0,87 \mathrm{eV}$. Ces deux valeurs sont faibles par rapport à l'énergie d'activation mesurée par Eisenstadt [8] : 1,1 eV. Nous attribuons cet effet à l'interaction covalente existant entre un centre $\alpha$ et un centre $F$ (ou un centre $\alpha$ et un centre $F_{2}$ ) suffisamment proches l'un de l'autre [9] : la migration du centre $\alpha$ serait dans tous les cas une migration à courte distance. Il faut remarquer aussi que nos énergies d'activation sont surtout indicatives car elles sont mesurées dans un domaine de température assez étroit.

Lorsque les cristaux de fluorure de lithium sont irradiés à une température supérieure à $270^{\circ} \mathrm{K}$ : les centres $F_{2}^{+}$et $F_{2}, F_{3}^{+}$et $F_{3}$ apparaissent et leur nombre dépend de la température de l'irradiation : les courbes des figures 2 et 3 sont relatives à des cristaux irradiés

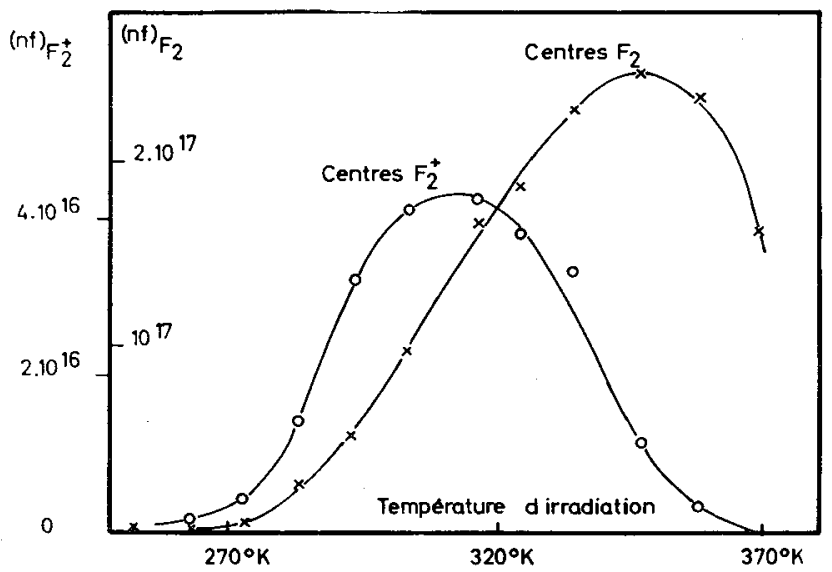

Fig. 2. - Variation avec la température d'irradiation du nombre de centres $F_{2}^{+}$et $F_{2}$ (aux forces d'oscillateur près) dans une série de cristaux de $\mathrm{LiF}$ irradiés aux électrons $(3 \times$ $10^{15} \mathrm{e} / \mathrm{cm}^{2} ; 1,4 \mathrm{MeV}$ ) à différentes températures (durée de chaque irradiation : 13 minutes).

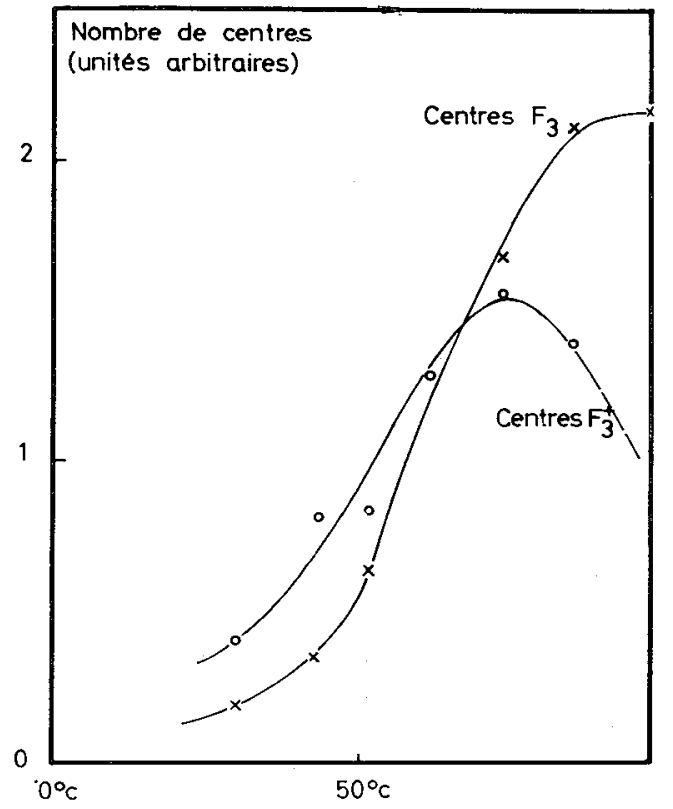

Fig. 3. - Variation avec la température d'irradiation du nombre de centres $F_{3}^{+}$et $F_{3}$ (valeurs relatives) dans une série de cristaux de LiF irradiés aux électrons $\left(3 \times 10^{15} \mathrm{e} / \mathrm{cm}^{2} ; 1,4 \mathrm{MeV}\right)$ à différentes températures (durée de chaque irradiation : 13 minutes).

aux électrons de $1,4 \mathrm{MeV}$ pendant $13 \mathrm{mn}\left(3 \times 10^{15}\right.$ $\left.\mathrm{e} / \mathrm{cm}^{2}\right)$ à des températures croissantes. Les centres $F_{2}^{+}$ et $F_{3}^{+}$apparaissent, cette fois encore, comme une étape 
intermédiaire à la formation des centres $F_{2}$ et $F_{3}$, et sont créés par diffusion de centres $\alpha$ vers les centres $F$ et $F_{2}$. Nous pouvons donc conclure que, dans le fluorure de lithium, les centres complexes se forment sous itradiation lorsque la température est supérieure à lambiante, il doit donc exister des centres $\alpha$ dans les cristaux à cette température.

II. Formation des centres $F$. - Les résultats obtenus pour la création des centres complexes nous ont amenés à reconsidérer les lois de création des centres $F:$ en effet des études cinétiques de formation des centres $F_{2}$ sous irradiation à la température ambiante montrent que le nombre de ces centres est une fonction linéaite de la dose d'irradiation (Fig. 4).

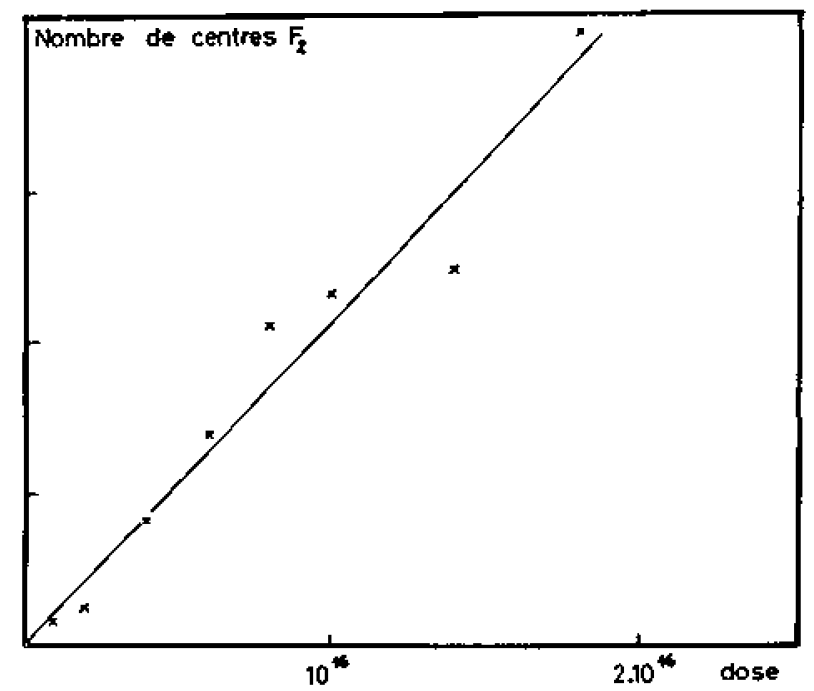

FIG. 4. - Variation du nombre de centres $F_{2}$ formés à la temperature ambiante en fonction de la dose d'électrons de 1,4 MeV (fiux égal à $1 \mu \mathrm{A} / \mathrm{cm}^{2}$ ).

Comme le nombre de centres $F_{2}$ croît comme le carré du nombre de centres $F$ [10] ces résultats suggéraient, pour le nombre de centres $F$ formés, une croissance proportionnelle à la racine de la dose. Les mesures effectuées jusqu'à présent avaient toujours donné pour les centres $F$ des lois sensiblement linéaires avec la dose [11], mais toujours dans le cas de concentrations faibles $\left(10^{-7}\right.$ a $\left.10^{-5}\right)$ et généralement à basse température. Nous avons done repris l'étude des cinétiques de création de centres $F$ pour des concentrations comprises entre $10^{-5}$ et $2 \times 10^{-4}$, la température d'irradiation etant comprise entre $77^{\circ} \mathrm{K}$ et $600^{\circ} \mathrm{K}$ et l'irradiation effectuée au moyen du faisceau de rayons $X$ non filtré d'une anode de cuivre $(42,5 \mathrm{kV}-8 \mathrm{~mA})$.

A la temperature ambiante, le nombre $n_{F}$, de centres
$F$, augmente avec le temps d'irradiation $t$ (Fig. 5) : si l'on reporte ce nombre en fonction de $\sqrt{t}$ on obtient une droite (Fig. 6). La création des centres $F$ à la température ambiante obéit donc à la loi : $n_{F}=k \sqrt{t}(3)$.

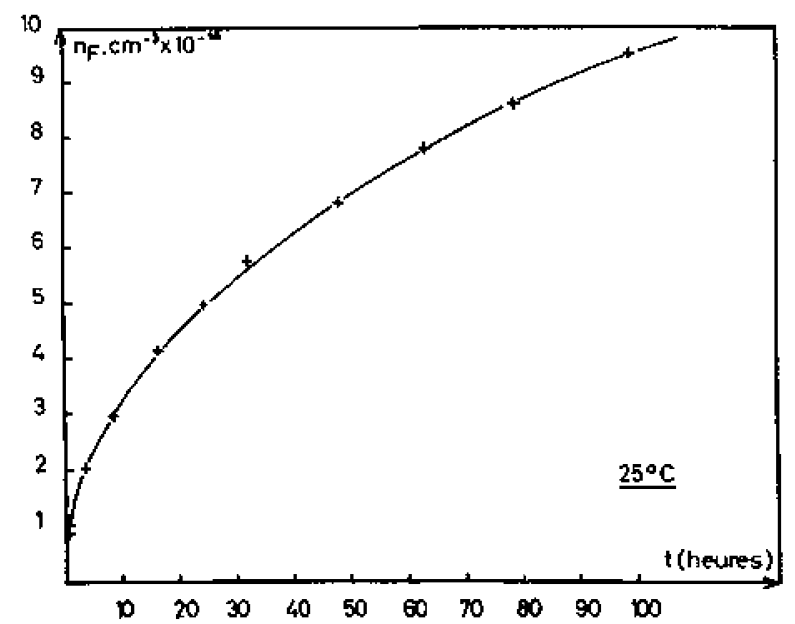

FtG. 5. - Variation du nombre de centres $F$ avec la duréc de l'irradiation à la température ambiante

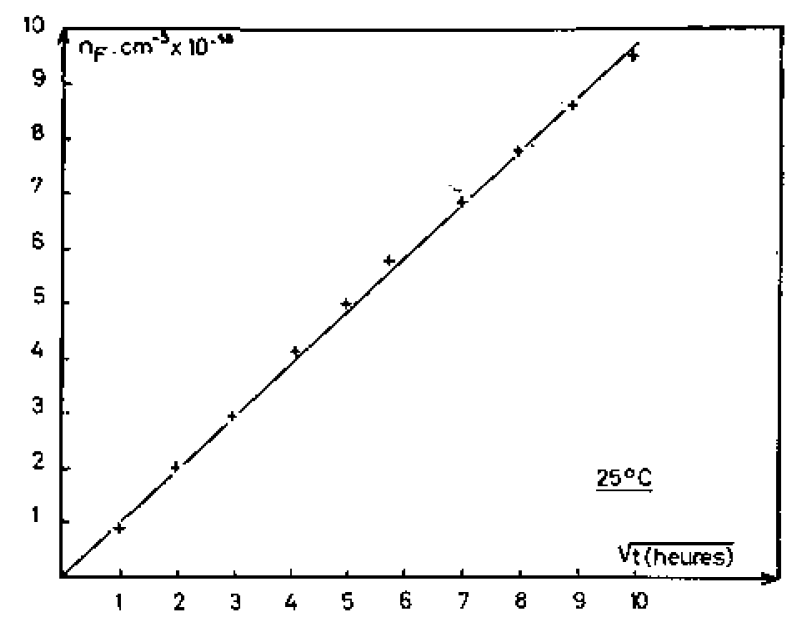

FiG. 6. - Variation du nombre de centres $F$ avec la racine carrée de la dose à la tempétature ambiante

Lorsque la température d'irradiation croît, la loi (3) est toujours observée, cependant la constante $k$, qui ne varje pas jusque $423^{\circ} \mathrm{K}$, décroît ensuite avec la température (Fig. 7 ).

Lorsque la température devient inférieure à l'ambiante, la loi de création à l'ambiante reste valable, pour les concentrations élevées, jusque $195^{\circ} \mathrm{K}$ (Fig. 8) bien que quelques écarts se manifestent pour les faibles concentrations. A $77 \circ \mathrm{K}$ cependant les résultats sont 


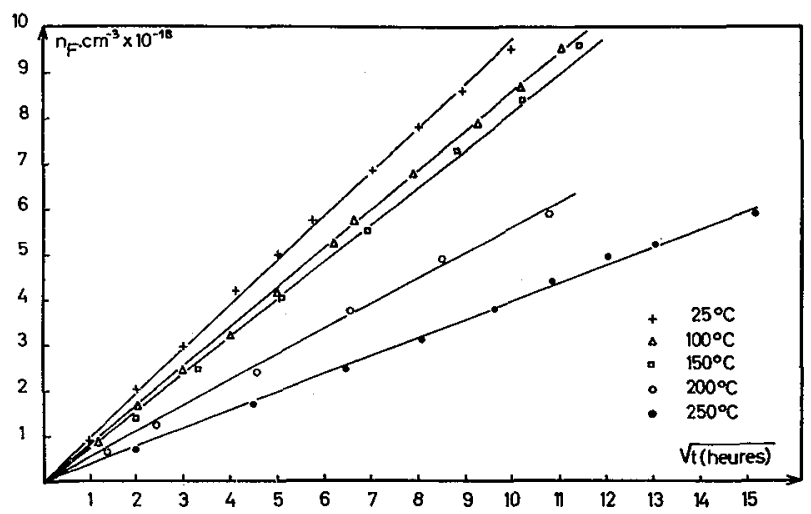

FIG. 7. - Courbes $n_{\mathrm{F}}=k \sqrt{t}$ aux températures supérieures à l'ambiante

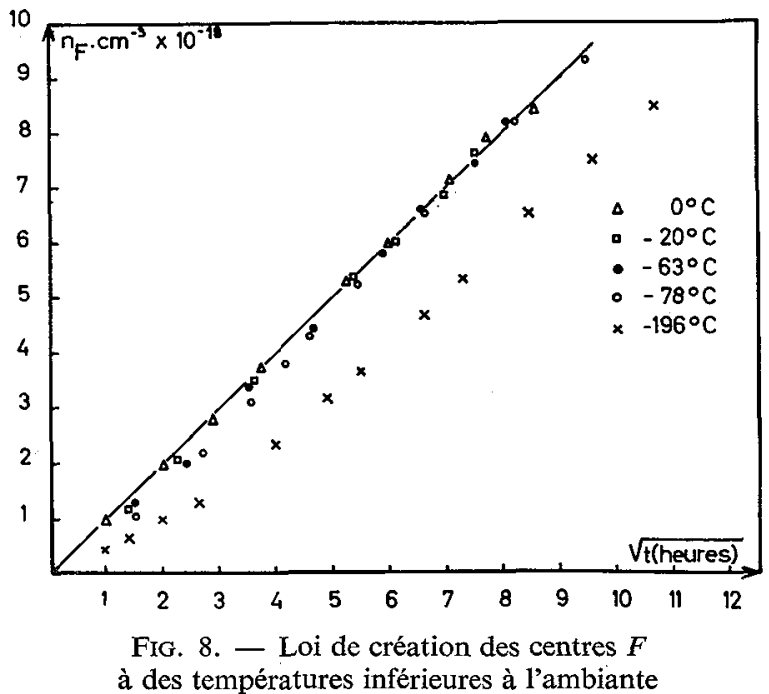

complètement différents bien que $n_{\mathrm{F}}$ semble toujours varier comme $\sqrt{t}$ pour les concentrations en centres $F$ élevées.

Ces résultats expérimentaux montrent donc que le nombre de centres $F$ formés est maximum autour de la température ambiante. Il varie comme la racine carrée de la dose lorsque la température d'irradiation est supérieure à $200^{\circ} \mathrm{K}$ et la concentration atomique comprise entre $10^{-5}$ et $2 \times 10^{-4}$. Certains auteurs ont proposé des mécanismes de formation conduisant à de telles lois de création : Goldstein [12], utilisant la théorie de Pooley [13] fait intervenir un mode de création des centres $F$ par désexcitation excitonique; Varley [14], reprenant le mécanisme de formation par ionisation multiple d'un ion halogène, prédit que l'éli- mination des interstitiels halogènes doit limiter le taux de formation des centres $F$. Ces deux théories se heurtent pour le moment à des difficultés. De toutes façons elles traduisent le fait que la vitesse de création des centres $F$ varie dans ce cas d'une manière inversement proportionnelle au nombre de centres $F$ lui-même.

Il faut remarquer de plus que, lorsque la température d'irradiation dépasse $423 \circ \mathrm{K}$, le taux de création des centres $F$ diminue et la formation des centres complexes est rapide. Dans ce cas la loi de formation des centres $F$ reste inchangée : $n_{\mathrm{F}}=k \sqrt{t}$, seule la constante $k$ diminue.

En conclusion nous avons pu interpréter les résultats expérimentaux concernant la formation des centres complexes d'une manière relativement simple car ils sont produits à partir des centres $F$ et les centres intermédiaires sont maintenant identifiés. En ce qui concerne les centres $F$ le problème est plus difficile et nous manquons de données expérimentales concernant la formation simultanée des autres centres : centres $\alpha$, halogènes interstitiels qui semblent présents dans les cristaux lors de l'irradiation à l'ambiante [15].

\section{Références}

[1] Schulman (J. H.) et Compton (W. D.), Color Centers in Solids, Pergamon Press, 1962.

[2] Mascarenhas (S.), Wiegand (D. A.) et SMoluCHOWski (R.), Phys. Rev., 1964, 134 A, 485.

[3] Kanzig (W.), J. Phys. Chem. Sol., 1960, 17, 88.

[4] FARGe (Y.), LAmbert (M.) et Smoluchowski (R.), Solid State Comm., 1966, 4, 333.

[5] Farge (Y.), Toulouse (G.) et Lambert (M.), $J$. Physique, 1966, 27, 287.

[6] Farge (Y.), Thèse Orsay, 1967.

[7] Delbece (C. J.), Z. Physik, 1963, 171, 560.

[8] Eisenstadt (M.), Phys. Rev., 1963, 132, 630.

[9] FaRge (Y.), LAMBERT (M.) et SMOLuChowsKi (R.), Phys. Rev., 1967, 159, 700.

[10] Compton (W. D.) et RABIN (H.), Solid State Physics, 1964, 16.

[11] Rabin (H.) et KLICK (C. C.), Phys. Rev., 1960, 117, 1005. Mitchell (P.), Wiegand (D. A.) et SMOluCHowski (R.), Phys. Rev., 1960, 117, 442. 1961, 121, 484.

[12] Goldstein (F. T.), Solid State Comm., 1966, 4, 621.

[13] Pooley (D.), Proc. Phys. Soc., 1966, 87, 245. 1966, $87,257$.

[14] Varley (J. H. O.), J. Phys. Chem. Solids, 1962, 23, 985.

[15] Peisl (H.), Batzer (R.) et Waidelich (W.), Phys. Rev. Letters, 1966, 17, 1129. Phys. Stat. Sol. 1966, 15, 495. 